\title{
Diversidad fúngica en el filoplano de Osmorhiza spp. relacionado con el sistema de regeneración empleado en bosques de Nothofagus pumilio en Tierra del Fuego, Argentina
}

Fungi diversity in Osmorhiza spp. phylloplane related to the regeneration system applied in Nothofagus pumilio forests in Tierra del Fuego, Argentina.

\author{
MARIA GABRIELA DUCID ${ }^{1}$, MONICA ADRIANA MURACE ${ }^{1}$, JUAN MANUEL CELLINI ${ }^{2}$ \\ ${ }^{1}$ Protección Forestal Fac. Cs. Agr. y Forestales, UNLP, La Plata, CP 1900, CC 31, Bs. As., Argentina. \\ E-mail: indforest1@ceres.agro.unlp.edu.ar \\ ${ }^{2}$ Biometría Forestal, Universidad Nacional de La Plata. La Plata, Buenos Aires, Argentina. \\ Centro Austral de Investigaciones Científicas, CC 92 (9410) Ushuaia, Tierra del Fuego, Argentina. \\ E-mail: jmc@ceres.agro.unlp.edu.ar
}

\begin{abstract}
SUMMARY
The aim of this work was to determine the diversity of the Osmorhiza chilensis phylloplane fungus in two regeneration systems to establish which of has the least impact on the diversity in a Nothofagus pumilio virgin forest in Tierra del Fuego. Forest management affects the biodiversity and equilibrium of the original system, altering different communities as, for instance, the one constituted by the phylloplane microflora. These changes were studied in two regeneration systems, shelterwood cut (CP) and clear cut (TR), against virgin forest (BV) as a standard. Leaves of $O$. chilensis were processed in a laboratory to isolate and identify fungi species. The parameters evaluated were the abundance, richness, and diversity indices. The Beta diversity indices showed that the communities that differed most were BV and TR and the most similar were BV and CP, which demonstrates that $\mathrm{CP}$ has the least impact on diversity. TR proved to be the most aggressive treatment, as it produced the maximum variation in relation to the type and number of species, which might be related to the loss of the entire protective cover.
\end{abstract}

Keywords: fungi species, phylloplane, forest management, diversity, conservation.

\section{RESUMEN}

El objetivo del trabajo fue determinar la diversidad fúngica del filoplano de Osmorhiza chilensis en dos sistemas de regeneración, para establecer cuál de ellos provoca el menor impacto sobre la diversidad del Bosque Virgen (BV) de Nothofagus pumilio en Tierra del Fuego. El aprovechamiento forestal de estos bosques afecta la biodiversidad y el equilibrio del sistema original, alterando las distintas comunidades, como la constituida por la micoflora del filoplano. Estos cambios se estudiaron en dos sistemas de regeneración: Corta de Protección (CP) y Tala Rasa (TR), con BV como testigo. Se trabajó con hojas de $O$. chilensis procesadas en laboratorio para aislar e identificar las especies fúngicas. Los parámetros evaluados fueron: abundancia, riqueza específica e índices de diversidad. TR presentó el menor valor de abundancia, la mayor riqueza y diversidad. Los índices de diversidad beta mostraron que las comunidades más diferentes fueron BV y TR y las más semejantes BV y $\mathrm{CP}$, lo cual demuestra que CP causa el menor impacto en la diversidad. TR resultó ser el más agresivo por provocar el mayor cambio en cuanto al tipo y número de especies, lo que estaría relacionado con la pérdida total de la cubierta protectora.

Palabras clave: especies fúngicas, filoplano, manejo forestal, diversidad, conservación. 


\section{INTRODUCCION}

En la República Argentina la lenga (Nothofagus pumilio (Poepp. et Endl.) Krasser) posee un amplio rango de distribución natural, desde los $36^{\circ} 50^{\prime}$ a $\operatorname{los} 55^{\circ} 02^{\prime} \operatorname{LS}(1,2)$. En la Patagonia argentina y chilena representa la masa forestal de mayor importancia en superficie y volumen. Ocupa cerca de tres millones de hectáreas, encontrándose el $27 \%$ en el sector argentino (3).

Estas formaciones boscosas tienen como finalidad principal producir bienes y servicios para la sociedad y generar empleo y en el sector argentino de Tierra del Fuego son usadas para pastoreo y el aprovechamiento forestal (4).

Se han propuesto numerosos sistemas para regenerar estos bosques $(2,5)$. Los más utilizados son la Corta de Protección (CP) y propuestas más modernas que incluyen diferentes grados y formas de retención. Estas últimas consisten en dejar un sector de bosque o agregado intacto por más de un turno, aplicando Tala Rasa (TR) al resto de la superficie (6). El tamaño de los agregados que se utilizan en Tierra del Fuego es de $30 \mathrm{~m}$ de radio, distribuidos homogéneamente a razón de uno por hectárea (7). La CP se basa en la cosecha gradual del dosel para provocar la regeneración y el desarrollo paulatino de un nuevo rodal bajo el dosel remanente (7). En la $T R$ todos los árboles se aprovechan antes de que ocurra la regeneración, dejando espacios totalmente abiertos (8).

En el sotobosque de estos bosques productivos la diversidad de especies en los ambientes cerrados está representada por los géneros $O s$ morhiza sp., Dysopsis sp., Viola sp., Adenocaulon sp., Galium sp., Blechnum sp. y Ranunculus sp. y por Cardamine sp., Gunnera sp., Senecio sp., Cotula sp., Rubus sp., Acaena sp. y Schizeilema $s p$. en los lugares abiertos y húmedos $(9,10)$. Además de especies nativas, existen numerosas especies exóticas con una amplia dispersión, por ejemplo: Cerastium fontanum Baumg., Rumex acetosella L., Veronica serpyllifolia L. y Taraxacum officinale Weber $(9,11,12)$. Sin embargo, el número de especies es bajo comparado con otros bosques templados del mundo $(9,10,13,14)$.

La diversidad de especies tiene componentes que responden a variaciones del ambiente. Uno de ellos es la riqueza, que es el número de especies de una comunidad por unidad de espacio. Otro componente de gran importancia es la abundancia (15).
En el análisis de una comunidad no basta con estos parámetros, sino que hay que tener en cuenta las relaciones entre especies, su distribución, la forma en que interaccionan, la influencia entre sí y con el medio físico que las rodea (16).

Las estructuras aéreas de las plantas, en particular las hojas, proporcionan un ambiente donde se desarrolla una variada flora microbiana, compuesta por organismos que pueden ser epífitos o endófitos, según su ubicación en el sustrato. Los epífitos forman parte del filoplano, siendo éste el hábitat que ocupan los organismos epífitos íntimamente relacionados con la hoja viva $(17,18)$. Estas comunidades son dinámicas en tiempo y espacio, variando en sus propiedades emergentes (diversidad, abundancia, competencias interespecíficas y cadenas tróficas) frente a cambios del ambiente, como la acción antrópica producida a través del uso del recurso forestal (16).

El objetivo del trabajo fue determinar la diversidad fúngica del filoplano de Osmorhiza chilensis en dos sistemas de regeneración: CP y TR para establecer cuál de ellos provoca el menor impacto sobre la diversidad del $B V$ de lenga en Tierra del Fuego.

\section{MATERIAL Y METODOS}

Se trabajó en un rodal de Nothofagus pumilio ubicado en la "Estancia San Justo", Tierra del Fuego, Argentina (54 06' LS, 68 $37^{\circ}$ LO). Este lugar pertenece fitogeográficamente a la Provincia Subantártica, en la que predominan el bosque caducifolio o perennifolio, praderas y turberas (1).

En esta zona el aserradero "Los Castores" realizó tareas de aprovechamiento en febrero de 2000 $(7,19)$, aplicando los sistemas de retención agregada y corta de protección. Se caracteriza por veranos cortos y fríos e inviernos largos, con presencia de nieve y heladas. Las temperaturas medias mensuales varían de -3 a $9^{\circ} \mathrm{C}$, con temperaturas medias por debajo de los $0^{\circ} \mathrm{C}$ durante tres meses. La precipitación anual oscila entre los 400 y $500 \mathrm{~mm}$ anuales (20).

Los datos climáticos de la época de muestreo (febrero de 2003) se detallan en el cuadro 1. En ellos se puede visualizar que el BV presenta mayor humedad relativa y menor precipitación que $\mathrm{CP}$ y TR, mientras que entre estos dos tratamientos las diferencias son mínimas. 


\section{CUADRO 1}

Variables climáticas de la zona de estudio para el mes de febrero de 2003

Climate variables in the zone under study, February 2003.

\begin{tabular}{|llccc|}
\hline & & $B V$ & $C P$ & $T R$ \\
\hline$P P M$ & $(\mathrm{~mm})$ & 10,00 & 25,00 & 23,80 \\
$H R P M(\%)$ & 72,47 & 68,79 & 68,37 \\
$T M M$ & $\left({ }^{\circ} \mathrm{C}\right)$ & 8,96 & 9,20 & 9,04 \\
$W$ & $(\mathrm{~km} / \mathrm{h})$ & 0,88 & 2,62 & 4,26 \\
\hline
\end{tabular}

BV: bosque virgen; CP: corta de protección; TR: tala rasa; PP M: Precipitación media mensual en mm; HR P M: Humedad relativa promedio mensual; T M M: Temperatura media mensual; W: Promedio de velocidad del viento.

El muestreo se realizó en dos áreas definidas por el tipo de aprovechamiento forestal: TR con cobertura del $0 \%$ y CP con una cobertura del dosel remanente del $52,1 \%$. A modo de testigo se utilizó el BV con una cobertura del 97,5\%. La superficie de cada tratamiento fue de 8 ha.

Para determinar la diversidad fúngica del filoplano se eligió a Osmorhiza chilensis Hooker et Arn. por ser característica del sotobosque de lenga (10) y encontrarse en las tres situaciones analizadas (9).

Se recolectaron 100 hojas maduras por tratamiento. Se consideró este estadio debido a que es el que presenta la mayor diversidad específica, posiblemente producto del estado nutricional de la hoja (18). Las muestras del tratamiento TR se tomaran dentro del componente TR de un sistema de retención agregada. El material se conservó a $4^{\circ} \mathrm{C}$ hasta su posterior procesamiento en el laboratorio.

De las hojas recolectadas se obtuvieron 300 discos (100 por tratamiento) de $6 \mathrm{~mm}$ de diámetro, con un sacabocados. Estos fueron lavados 25 veces por agitación continua durante 2 minutos. Las primeras 10 abluciones se hicieron con agua destilada estéril más detergente Tween 80 (una gota por litro) y los restantes con agua destilada estéril. Posteriormente, cada disco se cortó con un sacabocados de $2 \mathrm{~mm}$ de diámetro. Las submuestras obtenidas fueron colocadas en Cajas de Petri con papel de filtro estéril durante 24 horas para su secado $(21,22,23,24,25)$. Transcurrido este lapso se sembraron en Cajas de Petri con medio de cultivo Agar Extracto de Malta y se incubaron en estufa a $25^{\circ} \mathrm{C}$ durante 7 días para el aislamiento e identificación de las cepas fúngicas obtenidas.

Los indicadores de diversidad expresados por tratamiento fueron: a) abundancia, b) gráficos de solapamiento de especies y c) índices de diversidad alfa y beta.

La diversidad alfa se midió a través de la riqueza específica o $S$, el Indice de diversidad de Margalef $\left(D_{M g}\right)$, Indice de diversidad de Menhinick $\left(D_{M n}\right)$, Indice de Simpson $(1-\lambda)$ e Indice de Shannon $(\mathrm{H})$.

La diversidad beta se calculó a partir de los coeficientes de Jaccard para datos cualitativos y Sörensen para datos cuali y cuantitativos.

Para determinar la existencia de diferencias entre tratamientos se utilizaron tablas de contingencia. En su confección se tuvieron en cuenta sólo a las especies que presentaron abundancias mayores al $2 \%$. Además, se llevó a cabo un análisis multivariado de correspondencia por el método de los promedios recíprocos sobre datos de abundancia, con ponderación de las especies raras.

\section{RESULTADOS Y DISCUSION}

De las 300 muestras sembradas se aislaron 228 colonias fúngicas pertenecientes al filoplano. El número de colonias aisladas por tratamiento fue de: TR: 63 (28\%), CP: 82 (36\%) y BV: 83 (36\%). La abundancia disminuyó con la cobertura forestal, ya que BV y CP presentaron los valores más altos, descendiendo ésta en el tratamiento TR. En el resto del material no se observó desarrollo miceliar (cuadro 2).

De las colonias aisladas se identificaron 40 especies. El tratamiento testigo presentó el menor número con 10 especies y TR la mayor riqueza con 21 especies, mientras que CP presentó un valor intermedio. Del total (40) se determinó que 22 aparecieron sólo una vez en todo el ensayo. Cada una de ellas representó el 0,5\%. A pesar de su baja abundancia, fueron importantes en la determinación de las diferencias entre tratamientos. De estas 22, 11 de ellas se aislaron en TR, 10 en CP y 1 en BV. De las restantes especies, 5 presentaron una abundancia mayor al 5\% y de éstas, 2 cepas (GLO y MEH) fueron características de los tratamientos BV y TR, respectivamente. Para las 
3 restantes (BOC, CLH y NNP) se observó que su abundancia varió de acuerdo al tratamiento empleado (cuadro 3, figura 1). Si consideramos a las especies con abundancias mayores al $2 \%$ se incorporan a los tratamientos las cepas: CL2, MUC, HUM y AUR (figura 2).

\section{CUADRO 2}

Resumen de los resultados obtenidos del análisis de las muestras.

Summary of the sample analyses.

\begin{tabular}{|c|c|c|c|c|c|}
\hline Especie & Abrev. & $\mathrm{BV}$ & $\mathrm{CP}$ & $\mathrm{TR}$ & $\begin{array}{l}\text { Total } \\
\text { general }\end{array}$ \\
\hline Affinis Phoma & $A F F$ & & & 2 & 2 \\
\hline Aspergillus sp. Micheli ex Fries & $A S P$ & & & 1 & 1 \\
\hline Aureobasidium sp. Viala y G. Boyer & AUR & 3 & 3 & & 6 \\
\hline Botritys cinerea (Botriotinia) Pers ex Pers & $B O C$ & 4 & 12 & 2 & 18 \\
\hline Cladosporium cladosporioides (Fresen.) de Vries & $C L C$ & 1 & 1 & & 2 \\
\hline Cladosporium herbarum (Pers.) Link ex S. F. Gray & $\mathrm{CLH}$ & 3 & 21 & 10 & 34 \\
\hline Cladosporium sp. Link & $C L S$ & & 1 & & 1 \\
\hline Cladosporium sp. Link 1 & CL1 & & & 1 & 1 \\
\hline Cladosporium sp. Link 2 & $C L 2$ & & & 6 & 6 \\
\hline Chaetomium globosum Kuntze & $\mathrm{CHG}$ & & & 2 & 2 \\
\hline Fusarium sp. Link 1 & FUS & 1 & & & 1 \\
\hline Fusarium sp. Link 2 & FUL & 3 & & & 3 \\
\hline Gloeosporium sp. & $G L O$ & 25 & & & 25 \\
\hline Sphaeropsidal 1 & $H E 1$ & & & 2 & 2 \\
\hline Sphaeropsidal 2 & $H E 2$ & & 1 & & 1 \\
\hline Humícola grisea Traaen & $H U M$ & & 7 & & 7 \\
\hline Levadura & $L E V$ & & 1 & 3 & 4 \\
\hline Micelio Dematiáceo Estéril 1 & $M D 1$ & & & 1 & 1 \\
\hline Micelio Dematiáceo Estéril 2 & $M D 2$ & & 1 & & 1 \\
\hline Micelio Dematiáceo Estéril 3 & $M D 3$ & & & 1 & 1 \\
\hline Micelio Dematiáceo Estéril 4 & MD4 & 1 & & 1 & 2 \\
\hline Micelio Estéril 1 & $M E$ & & & 1 & 1 \\
\hline Micelio Estéril 2 & $M E H$ & & & 21 & 21 \\
\hline Monilia sp. Pers & $M O N$ & & & 1 & 1 \\
\hline Mucor sp. Micheli & $M U C$ & & 8 & & 8 \\
\hline Micelio no identificado & $N N$ & & 1 & & 1 \\
\hline Micelio no identificado 1 & NN 1 & & 1 & 3 & 4 \\
\hline Micelio no identificado 2 & NN 2 & & & 1 & 1 \\
\hline Micelio no identificado 3 & NN 3 & & 1 & & 1 \\
\hline Micelio no identificado 4 & NN 4 & & 1 & & 1 \\
\hline Micelio no identificado 5 & NN 5 & & 1 & & 1 \\
\hline Micelio no identificado 6 & NN 6 & & 1 & & 1 \\
\hline Micelio no identificado 7 & NN 7 & & 1 & & 1 \\
\hline Micelio no identificado 8 & $N N 8$ & & 1 & & 1 \\
\hline Micelio no identificado 9 & NN 9 & & & 1 & 1 \\
\hline Micelio no identificado 10 & NN 10 & & & 1 & 1 \\
\hline Micelio no identificado 11 & NN 11 & & & 1 & 1 \\
\hline Micelio no identificado 12 & NN 12 & & & 1 & 1 \\
\hline Micelio no identificado MP & $N N P$ & 39 & 16 & & 55 \\
\hline Penicillium sp. Link & PEN & 3 & 2 & & 5 \\
\hline \multirow[t]{2}{*}{ Sin presencia de micelio } & $N A D$ & 17 & 18 & 37 & 72 \\
\hline & & 100 & 100 & 100 & 300 \\
\hline
\end{tabular}

Abrev.: abreviatura de la denominación; CP: tratamiento de corta de protección; TR: tratamiento de tala rasa; BV: bosque virgen (testigo). 


\section{CUADRO 3}

Especies con abundancias mayores al 5\% del total. Species with abundance greater than $5 \%$ of the total.

\begin{tabular}{|lrrrrr|}
\hline Tratamiento & BOC & CLH & GLO & MEH & NNP \\
\hline BV & 4 & 3 & 25 & & 39 \\
CP & 12 & 21 & & & 16 \\
TR & 2 & 10 & & 21 & \\
Total & 18 & 34 & 25 & 21 & 55 \\
\hline
\end{tabular}

CP: tratamiento de corta de protección; TR: tratamiento de tala rasa; BV: bosque virgen (testigo); BOC: Botritys cinerea (Botriotinia); CLH: Cladosporium herbarum; GLO: Gloeosporium sp.; MEH: Micelio estéril 2; NNP: Micelio no identificado tipo MP.

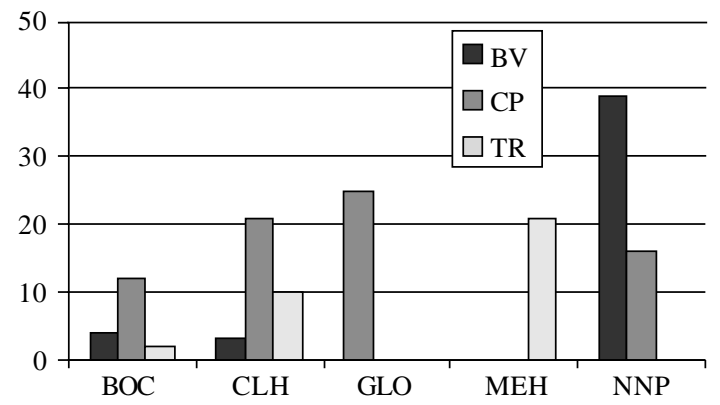

CP: tratamiento de corta de protección; TR: tratamiento de tala rasa; BV: bosque virgen (testigo); BOC: Botritys cinerea (Botriotinia); CLH: Cladosporium herbarum; GLO: Gloeosporium sp.; MEH: Micelio estéril 2; NNP: Micelio no identificado tipo MP.

Figura 1. Porcentaje de colonias fúngicas observadas por especie en cada uno de los tratamientos ensayados con abundancia mayor al $5 \%$.

Percentage of fungi colonies observed by species in each of the tested treatment areas with an abundance greater than $5 \%$.

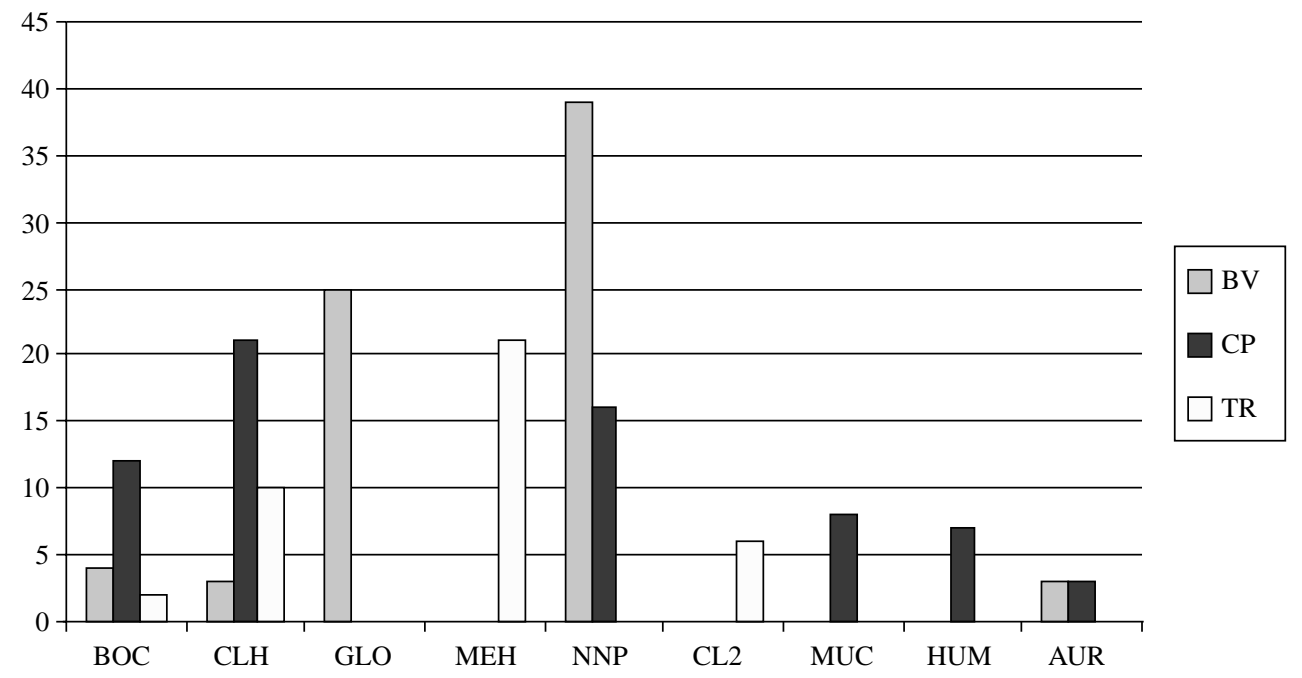

CP: tratamiento de corta de protección; TR: tratamiento de tala rasa; BV: bosque virgen (testigo); BOC: Botritys cinerea (Botriotinia); CLH: Cladosporium herbarum; GLO: Gloeosporium sp.; MEH: Micelio estéril 2; NNP: Micelio no identificado tipo MP; CL2: Cladosporium sp. 2, HUM: Humícola grisea; AUR: Aureobasidium sp.; NNP: Micelio no identificado tipo MP, PEN: Penicillium sp.

Figura 2. Porcentaje de colonias fúngicas observadas por especie en cada uno de los tratamientos ensayados con abundancia mayor al $2 \%$.

Percentage of fungi colonies observed by species in each of the tested treatments with abundance an greater than $2 \%$.

Análisis de solapamiento: Mediante los análisis de solapamientos se observó un bajo porcentaje de especies comunes entre BV y TR (MD4, BOC y CLH) y CP y TR (BOC, CLH,
LEV, NN1). En BV y CP se encontró el mayor porcentaje de especies compartidas. Estas fueron: BOC, CLH, AUR, CLC, NNP y PEN (figura 3). 


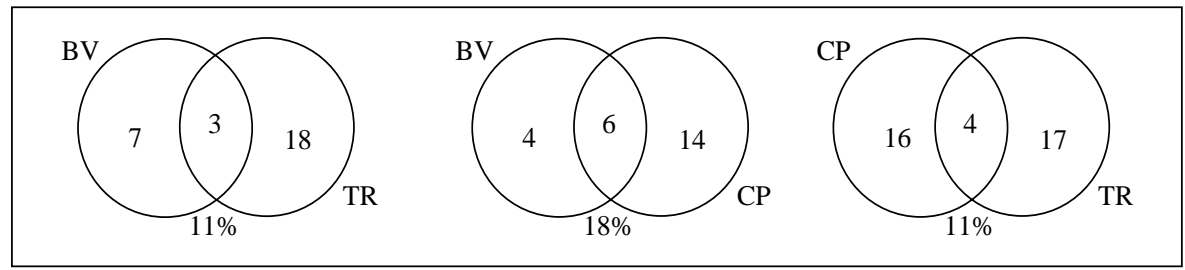

CP: corta de protección, TR: tala rasa y BV: bosque virgen.

Figura 3. Superposición de especies fúngicas entre tratamientos ensayados. Overlap of fungi species between tested treatment areas.

En la figura 4 se observan los solapamientos de las tres situaciones analizadas. Solo dos especies fueron comunes a los tres tratamientos: BOC y CLH. Asimismo en TR se identificaron 18 nuevas especies respecto del BV. Estas se presentaron con bajas abundancias (menores al 5\%), excepto dos: MEH y CL2, para las que se determinó una abundancia del $33,33 \%$ y $9,5 \%$, respectivamente. Las especies exclusivas de este tratamiento fueron 16 en total: AFF, ASP; CL1; CL2; CHG, HE1, MD1, MD3, ME, MON y 5 especies no identificadas.

CP incorporó 14 especies nuevas, donde sólo se destacaron por su abundancia MUC. y HUM (con 9,75 y $8,5 \%$, respectivamente, sobre el total del tratamiento). Para el resto de las especies se determinaron abundancias inferiores al 1,5\%.

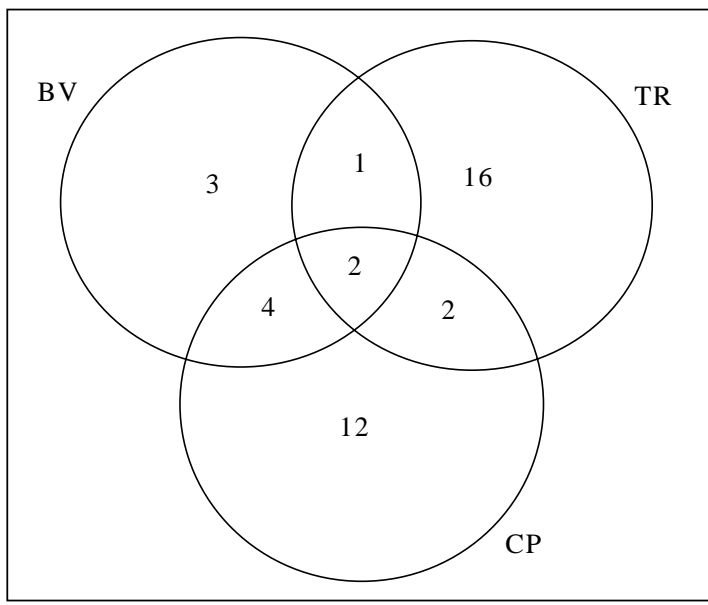

$\mathrm{CP}$ : corta de protección, TR: tala rasa y $\mathrm{BV}$ : bosque virgen.

Figura 4: Superposición de especies fúngicas entre los tratamientos ensayados considerando al $100 \%$ de las especies.

Overlap of fungi species between tested treatment areas, considering $100 \%$ of the species.
Al considerar para el análisis de solapamiento a las especies que se presentaron con una abundancia mayor al $2 \%$ (figura 5), se observó que las especies exclusivas se redujeron en todos los tratamientos. En TR y CP se determinaron 2 (MEH y CL2 en TR y MUC y HUM en CP). BV presentó 1 especie exclusiva (GLO) y compartió con CP 4 especies: AUR, NNP, BOC y CLH. Las dos últimas compartidas también con TR.

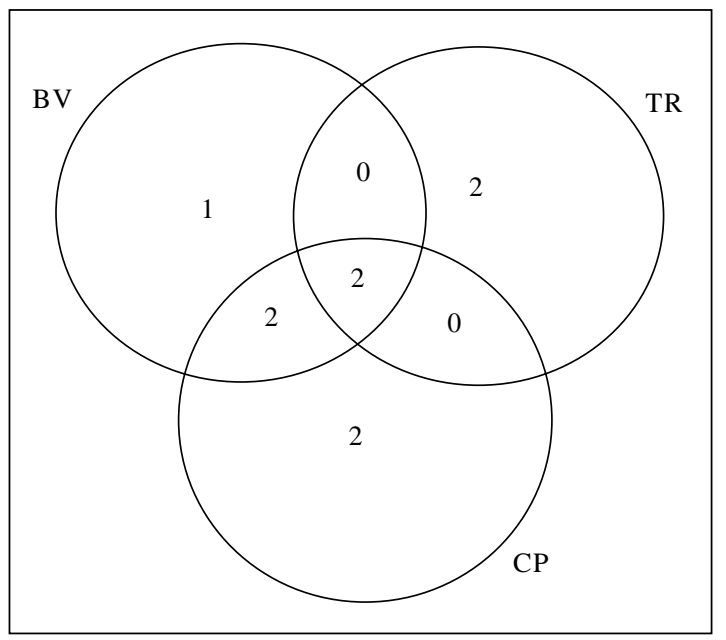

$\mathrm{CP}$ : corta de protección, TR: tala rasa y BV: bosque virgen.

Figura 5. Superposición de especies fúngicas en los tratamientos ensayados considerando a las abundancias mayores a $2 \%$.

Overlap of fungi species in the tested treatments considering abundance greater than $2 \%$.

Cuando se tuvo en cuenta la superposición de especies, con abundancias mayores al $5 \%$ entre tratamientos, se determinó que sólo una especie fue exclusiva para BV (GLO) y otra para TR (MEH). 
(figura 6) Las especies comunes a los 3 tratamientos fueron las determinadas en los análisis de solapamiento anteriores.

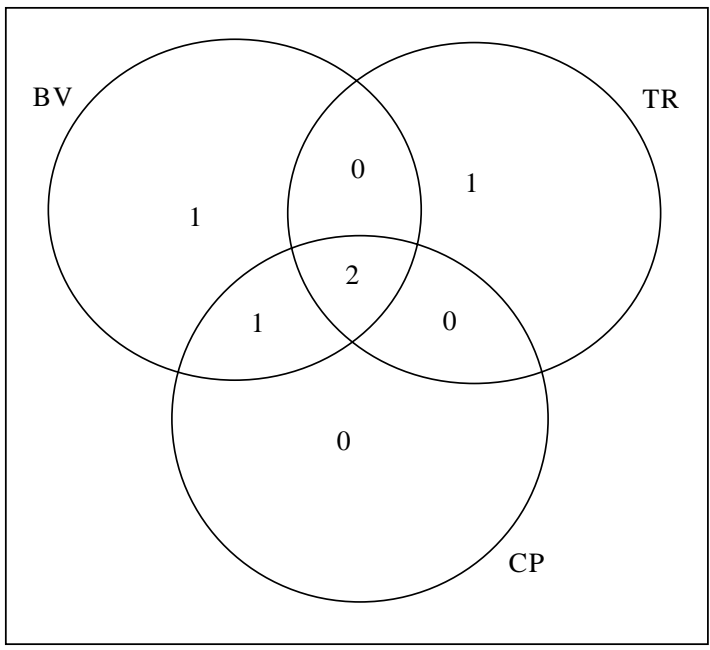

CP: corta de protección, TR: tala rasa y BV: bosque virgen.

Figura 6. Superposición de especies fúngicas en los tratamientos ensayados considerando a las abundancias mayores a $5 \%$.

Overlap of fungi species in the tested treatments considering abundance greater than $5 \%$.

Diversidad $\alpha$ (alfa). Al comparar los tratamientos mediante índices de diversidad alfa se observó un comportamiento diferente entre los mismos, originado en la forma de cálculo de cada uno de ellos (19). Estos índices manifestaron, en general, que los tratamientos $\mathrm{CP}$ y TR tuvieron un ambiente más diverso que el $B V$. El tratamiento TR fue el de mayor diversidad. El índice de Margalef marcó a TR como el tratamiento con más especies por muestra en proporción al total, con un valor de 4,8273. El índice más bajo se encontró en BV, con 2,0367. El tratamiento $\mathrm{CP}$ dio un valor intermedio con 4,3116. El índice de Menhinick marcó las mismas diferencias entre tratamientos. Los datos obtenidos mediante Shannon-Weaver aportaron resultados similares a los obtenidos (cuadro 4).

Simpson fue el único índice de diversidad alfa que aportó datos diferentes a los ya obtenidos, ya que mostró a $\mathrm{BV}$ como al tratamiento de mayor diversidad. Esto ocurre debido a que este índice le resta importancia a aquellas especies que aparecen con baja abundancia (25).

\section{CUADRO 4}

Riqueza e índices de diversidad alfa de los tratamientos estudiados.

Richness and alpha diversity indices of the treatments under study.

\begin{tabular}{|llll|}
\hline Índice & \multicolumn{1}{c}{$\mathrm{BV}$} & \multicolumn{1}{c}{$\mathrm{CP}$} & \multicolumn{1}{c|}{$\mathrm{TR}$} \\
\hline $\mathrm{N}$ & 83 & 82 & 63 \\
$\mathrm{~S}$ & 10 & 20 & 21 \\
$\mathrm{D}_{\mathrm{Mg}}$ & 2,0367 & 4,3116 & 4,8273 \\
$\mathrm{D}_{\mathrm{Mn}}$ & 1,0976 & 2,2086 & 2,6458 \\
$1-\lambda$ & 0,9026 & 0,8932 & 0,8433 \\
$\mathrm{H}$ & 0,6524 & 0,9973 & 1,0421 \\
\hline
\end{tabular}

$\mathrm{N}$ : Número total de colonias o abundancia total; S: Riqueza específica; $\mathrm{D}_{\mathrm{Mg}}$ : Indice de Margalef; $\mathrm{D}_{\mathrm{Mn}}$ : Indice de Menhinick; $H$ : Indice de Shannon-Weaver; $1-\lambda$ : Indice de Simpson; valores más altos del índice, mayor diversidad del tratamiento; $\mathrm{BV}$ : bosque virgen; CP: corta de protección; TR: tala rasa.

Diversidad $\beta$ (beta). Al comparar tratamientos mediante el Indice de Sörensen Cualitativo se determinó que TR fue el ambiente más disímil a BV. En contraposición, BV y CP fueron los tratamientos que presentaron más similitud. Los Indices de Sörensen cuantitativo y el de Jaccard, mostraron a BV y TR como los tratamientos más diferentes. Las mayores semejanzas se presentaron, al igual que con Sörensen Cualitativo, entre BV y CP. También se observó que los valores intermedios de los índices de diversidad fueron entre los tratamientos CP y TR, presentando valores cercanos a BV y TR. Todos los índices beta coincidieron en sus resultados, llevando por lo tanto a la misma conclusión en todos los casos (cuadro 5).

Análisis de cluster: En este análisis, la unión de los distintos tratamientos en conjuntos o bloques se interpreta como que dichos tratamientos son similares o más afines en cuanto a su composición de especies. La distancia horizontal indica un valor de similitud de los tratamientos. CP y TR fueron los ambientes que constituyeron la relación más cerrada, conformando un grupo aparte de BV. Esto manifestó similitud entre ambos tratamientos. 


\section{CUADRO 5}

Indices de diversidad beta entre los tratamientos ensayados.

Beta diversity indices between tested treatments.

\begin{tabular}{|lcccccccc|}
\hline Sörensen Cualit. & BV & TR & Sörensen Cuant. & BV & TR & Jaccard & BV & TR \\
\hline CP & 0,4 & 0,2 & CP & 0,35 & 0,19 & CP & 0,25 & 0,1081 \\
TR & 0,19 & - & TR & 0,08 & - & TR & 0,1071 & - \\
\hline
\end{tabular}

BV: bosque virgen; CP: corta de protección; TR: tala rasa; Cuant.: cuantitativo; Cualit.: cualitativo. Valores cercanos a 1, similitud entre tratamientos.

Cuadros de contingencia: En todos los análisis realizados el $\mathrm{P}$ Value fue significativo, denotando diferencias entre los tratamientos ensayados. En el cuadro 6 se pueden observar los resultados de las comparaciones entre los tratamientos. El Lambda indicó que la mayor asociación se dio en los tratamientos TR - BV, dando también el mayor valor de $\mathrm{Chi}^{2}$.

\section{CUADRO 6}

Tabla de Contingencia, teniendo en cuenta aquellas especies que presentaron abundancias mayores al $2 \%$.

Contingency table, considering species with abundance greater than $2 \%$.

\begin{tabular}{|lccc|}
\hline & P valor & Lambda & Chi cuadrado \\
\hline CP-TR & 0,0000 & 0,2878 & 63,88 \\
CP-BV & 0,0000 & 0,2732 & 77,00 \\
TR-BV & 0,0000 & 0,4242 & 100,08 \\
\hline
\end{tabular}

En el caso de la interacción entre los tres tratamientos, el valor de Lambda fue intermedio y no distinguió entre el uso del 2 y $5 \%$ de la muestra. El P Value arrojó diferencias entre los distintos tratamientos, al igual que el $\mathrm{Chi}^{2}$. Este último dio valores muy altos (cuadro 7).

Una característica de las comunidades es que contienen unas pocas especies que aparecen con altas abundancias y un gran número de especies que son raras y presentan bajas abundancias (25, 28), lo que se comprueba en este trabajo a través de los resultados obtenidos. En los tratamientos aparecen una o más especies dominantes sobre el

\section{CUADRO 7}

Interacción BV - CP - TR.

$B V-C P-T R$ interaction.

\begin{tabular}{|lccc|}
\hline Abundancia & P valor & Lambda & Chi cuadrado \\
\hline$>5 \%$ & 0,0000 & 0,3406 & 189,29 \\
$>2 \%$ & 0,0000 & 0,3500 & 221,36 \\
\hline
\end{tabular}

resto. Para BV estas especies fueron NNP y GLO ( $47 \%$ y $30 \%$, respectivamente, del total de la abundancia del tratamiento). En CP las especies dominantes fueron: NNP $(19,5 \%)$ y CLH $(25,6 \%)$. Los tratamientos CP y BV compartieron una especie que fue dominante en ambos, lo que los acerca en sus similitudes (cuadro 3). En TR se determinó como cepa dominante y exclusiva a MEH, hecho que marcó diferencia con los tratamientos $\mathrm{BV}$ y CP. Las especies acompañantes de la dominante en TR presentaron bajas abundancias y la mayoría de ellas fueron también exclusivas de este tratamiento. De todas las especies que este tratamiento compartió con $\mathrm{BV}$ y CP sólo una resultó dominante en otro tratamiento: $\mathrm{CLH}$ en CP. Esto remarcó las bajas similitudes entre BV y TR, tratamientos que no compartieron ninguna especie que haya sido dominante en ambos o sólo en alguno de ellos.

Con respecto a la abundancia TR presentó el menor valor. Esto podría estar relacionado con las condiciones más adversas que se presentan para el desarrollo fúngico, provocadas por la desaparición del dosel, el cual actúa como cubierta protectora. Este fenómeno se produce porque el patrón de colonización fúngica es determinado en gran medida por las condiciones ambientales (27). 
Según Venedikian (23), los principales factores que determinan la distribución de los hongos en las hojas son la topografía de la misma y la radiación solar. Esta última parece limitar el crecimiento miceliar, que es menor en las superficies más expuestas, lo que podría ser la causa de la disminución de la abundancia en el tratamiento de TR.

En cuanto a la diversidad, según los análisis realizados, ésta presenta valores máximos en TR, seguida por $\mathrm{CP}$ y finalmente por $\mathrm{BV}$ con los valores más bajos. De acuerdo con los datos registrados aumentaría con la disminución de la humedad ambiental (mayor en BV). Esto no coincide con otros autores, quienes determinaron cambios en la diversidad y abundancias fúngicas a través de las estaciones climáticas: Lee y Hyde (26) concluyeron que a más humedad en los ambientes mayores son las abundancias y riqueza de especies. Según Cabral (24) existe una correlación directa entre la abundancia y la humedad del ambiente e inversa entre la abundancia y la temperatura, mientras que la diversidad fúngica del filoplano no sufre variaciones con los cambios climáticos estacionales. Esto se cumple en lo que respecta a abundancias, pero no coincide con lo determinado al evaluar diversidad.

Los factores climáticos mencionados no presentaron grandes diferencias entre tratamientos. No ocurrió lo mismo con la precipitación media mensual (se duplica en ambos tratamientos respecto de BV) y la velocidad del viento (máxima en TR, intermedia en CP y mínima en BV) (cuadro 1). A medida que estas variables se incrementaron aumentó la diversidad y disminuyeron las abundancias.

Para explicar la diversidad máxima en TR, se debe tener en cuenta que si las comunidades existiesen en un equilibrio continuo y estuviesen en sitios espacialmente uniformes, la exclusión por competencia entre especies daría como resultado comunidades dominadas por pocas especies: las mejores competidoras (28). Esto no ocurre, ya que muchos factores impiden este equilibrio: predación, herbívoros, fluctuaciones en los factores físicos, catástrofes; todos ellos disturbios. Si éstos ocurren de manera continua, las primeras especies en extinguirse resultan ser aquellas que poseen tasas de crecimiento bajas con respecto al resto. Si los disturbios son raros, el sistema llega a un equilibrio competitivo, en donde las especies con menor capacidad de competitividad se pierden. Entre estos dos extremos existe un nivel intermedio de distur- bio, en el que la biodiversidad es máxima. Esta idea se denomina Hipótesis del Disturbio Intermedio (28). Los disturbios también operan a nivel de escala local, creando sectores que experimentan sucesión, caracterizado cada uno por una determinada composición de especies, la cual varía de uno a otro, pero que a gran escala se mantiene constante, incluyendo desde las especies pioneras hasta las pertenecientes al clímax (28).

Todas estas observaciones permiten afirmar que existen diferencias entre tratamientos en lo que respecta a la diversidad fúngica del filoplano de Osmorhiza sp. Estas se incrementan en función del grado de alteración que se provoque en el BV. Las características del BV se ven modificadas en forma más acentuada cuando se aplica TR en vez de CP. Esto ocurre porque la CP produce cambios más leves en el ambiente que los provocados por TR, lo que da como resultado una comunidad más similar a la del BV.

\section{CONCLUSIONES}

Las especies fúngicas del filoplano se ven significativamente afectadas cuando el bosque se interviene mediante CP y TR. En ambos casos, las condiciones ecológicas son modificadas, perjudicando a especies residentes del bosque virgen y propiciando la aparición de nuevas especies. El perjuicio, en algunos casos, se refleja a través de la disminución de la abundancia de especies, mientras que en otros causa la pérdida total de las mismas. Con la intervención, se pierden entre un 40 y $70 \%$ de las 10 especies originales del BV (CP y TR, respectivamente), a la vez que surgen otras nuevas, duplicándose la riqueza. Esto se manifiesta de manera más notoria en el caso de TR, posiblemente por ser éste el tratamiento que tiene mayor impacto en las características del BV. Al realizar TR, la cobertura se reduce drásticamente de un $97,5 \%(\mathrm{BV})$ al $0 \%$. En CP se genera una situación intermedia con un dosel remanente del $52,1 \%$. Como consecuencia de las diferentes cubiertas, las variables climáticas cambian. La precipitación media mensual (febrero) aumenta de $10 \mathrm{~mm}$ a $25 \mathrm{~mm}$ en CP y a $23,8 \mathrm{~mm}$ en TR. La humedad relativa mensual del ambiente tiende a descender junto con la cobertura, al contrario de lo que sucede con la velocidad del viento, que se incrementa de 0,88 a $4,26 \mathrm{~km} / \mathrm{h}$. Estos cambios en 
el ambiente son los responsables de las variaciones en la diversidad y abundancia fúngicas. Si bien en los dos tratamientos hay pérdida e incorporación de especies y variaciones en la abundancia, el tratamiento CP conserva especies dominantes del BV, a diferencia de TR, en donde el impacto es mayor y éstas se pierden. El mantenimiento de la diversidad del sistema original debe ser uno de los objetivos prioritarios, debiéndose aplicar métodos de aprovechamiento que lo modifiquen en la menor medida posible.

\section{AGRADECIMIENTOS}

Deseamos expresar nuestro agradecimiento por la ayuda desinteresada en la corrección del manuscrito a María Vanesa Lencinas, Nadia Salim y a Guillermo Martínez Pastur. A Carolina I. Llavallol, por la traducción del resumen, y a Guillermo Gea Izquierdo y Alicia Moretto por la recolección del material de muestreo. Finalmente, agradecemos en forma especial las sugerencias realizadas por el Dr. Marcelo Barrera.

\section{BIBLIOGRAFIA}

(1) CABRERA, A. L. Fitogeografía de la República Argentina. Bol. Soc. Argent. Bot., 1971, vol. 14, No 1-2, p. 1-42.

(2) MARTÍNEZ PASTUR, G., J. CELLINI, P. PERI, R. VUKASOVIC, C. FERNÁNDEZ. Timber production of Nothofagus pumilio (Poepp et Endl.) Krasser forests by a shelter wood system in Tierra del Fuego (Argentina). Forest Ecology and Management, 2000, $\mathrm{N}^{\circ} 134$, p. 153-162.

(3) ALFONSO, J. Los bosques de Tierra del Fuego. Revista Suelo Argentino, 1942, $\mathrm{N}^{\circ}$ 1, p. 47-51.

(4) BAVA, J. Y P. BURSCHEL. El Manejo de la Lenga. CIEFAP. Esquel, Argentina. Folleto de Divulgación, $1999, \mathrm{~N}^{\circ} 12,25 \mathrm{p}$.

(5) SCHMIDT, H., A. URZUA. Transformación y Manejo en los Bosques de Lenga en Magallanes. Departamento de Silvicultura y Manejo. Facultad de Ciencias Agrarias, Veterinarias y Forestales, Universidad de Chile. Santiago, Chile. Ciencias Agrícolas, 1982, № 11, 62 p.

(6) FRANKLIN, J., D. BERG, D. THORNBURGH, J. TAPPEINER. Alternative silvicultural approaches to timber harvesting: Variable retention harvest systems. En: Kohm, K. y Franklin, J. (Eds.). Creating a Forestry for the $21^{\text {st }}$ Century. Island press, 1997, p. 111-140.

(7) CELLINI, J. M., G. MARTÍNEZ PASTUR, R. VICENTE, B. DÍAZ, E. WALTER, M. BROUVER, R. VUKASOVIC. Rendimiento y calidad de trozas de diferentes sistemas de regeneración con retención dispersa y agregada, floreo, corta de protección y tala rasa aplicados en bosques de Nothofagus pumilio. Actas $1^{\text {er }}$ Congreso Chileno de Ciencias Forestales. Santiago de Chile, Chile. 23-25 octubre 2002, 44 p.
(8) DANIEL, T. W., J. A. HELMS, F. S. BACKER. Principios de la Silvicultura. $1^{\mathrm{a}}$ ed. en español, México: Mc Graw-Hill. Cáp. 18, Métodos de Reproducción, 1982, p. 427-446.

(9) MARTÍNEZ PASTUR, G., P. L. PERI, C. FERNÁNDEZ, G. STAFFIERI, M. V. LENCINAS. Change in understory species diversity during the Nothofagus pumilio (Poepp et Endl.) Krasser forest management cycle. J. For. Res, 2002, № 7, p. 165-174.

(10) MOORE, D. Flora of Tierra del Fuego. Anthony Nelson-Missouri Botanical Garden, 1983, p. 395.

(11) MOORE, D. M, R. N. GOODALL. La flora adventicia de tierra del Fuego. An. Inst. Patag. Punta Arenas, 1977, No 8, p. 263-274.

(12) COLLANTES, M.B., J. ANCHORENA. Las malezas exóticas y plantas escapadas de cultivos en la región de estepa de Tierra del Fuego. Parodiana, 1993, No 8, p. 213-217

(13) CHRISTENSEN, M., J. EMBORG. Biodiversity in natural versus managed forest in Denmark. For. Ecol. Manage, 1996, $\mathrm{N}^{\circ} 85$, p. 47-51.

(14) LIU, Q., A. KONDOH, N. TAKEUCHI. The forest vegetation and its differentation under disturbance in a temperate mountain. China. J. For. Res, 1998, No 3, p. 111-411.

(15) ODUM, E. Ecología. 3ra ed. México: Interamericana. 1974, $639 \mathrm{p}$

(16) BEGON, M., J. L. HARPER y C. R. TOWNSEND. Ecology: individuals, populations and communities. 3ra ed. Oxford: Blackwell Science. 1996, 1.068 p.

(17) KERLING, L. C. P. De microflora up het blad von Beta vulgaris. Tijdschrift over Planteaziekten, 1958, $\mathrm{N}^{\circ} 64$, p. 402-410.

(18) VENEDEKIAN, N., A. M. GODEAS. Estudio de la filósfera de Pinus taeda (Pinaceae) I. Poblaciones Fúngicas. Bol. Soc. Argent. Bot., 1996, vol. 31, No 3-4, p. 193-200

(19) GALLO, E. Densidad y riqueza de especies de aves en bosques productivos de Nothofagus pumilio (Poepp. et Endl.) Krasser con y sin intervención silvícola en Tierra del Fuego. Tesina de grado de la Universidad Nacional del Litoral, Santa Fe, 2003, 107 p.

(20) CUEVAS, J. Tree recruitment at the Nothofagus pumilio (Poepp et Endl.) Krasser alpine timberline in Tierra del Fuego Chile. J. Ecol., 2000, No 84, p. 47-51.

(21) KENDRICK, W. B., A. BURGES. Biological aspects of the decay of Pinus sylvestris leaf litter. Nova Hedwigia, 1962, IV(3-4), p. 313-342.

(22) WILDMAN H. G., D. PARKINSON. Microfungal succession on living leaves of Populus tremuloides. Can. J. Bot., 1978 , vol. 57 , p. $2.800-2.811$.

(23) VENEDEKIAN, N. Análisis de la Micoflora en hoja viva de Pinus taeda. PHYSIS (Buenos Aires), Secc. C, 1984, vol. 42, No 102, p. 7-16.

(24) CABRAL, D. Phyllosphere of Eucalyptus viminalis: Dynamics of fungal populations. Trans. Br. Mycol. Soc., 1985, vol. 85, No 3, p. 501-511.

(25) GODEAS, A. M. Estudios de descomposición en plantaciones de Pinus taeda III. Sucesión fúngica. Bol. Soc. Argent. Bot., 1992, vol. 28, No 1-4, p. 151-157.

(26) LEE, O. H. K., K. D. HYDE. Phylloplane fungi in Hong Kong mangroves: evaluation of study methods. The Mycological Society of America, Lawrence, 2002, vol. 94, No 4, p. 596-606.

(27) MISHRA, R. R., C. H. DICKINSON. Phylloplane and litter fungi of Ilex aquifolium. Trans. Br. Mycol. Soc., 1981, vol. 77, No 2, p. 329-337.

(28) KREBS, C. J. Ecology. The Experimental Analysis of Distribution and Abundance. $4^{\text {th }}$ ed. New York: Harper Collins. 1994. 801 p. 